\title{
Obesity Prevalence Among Adults Living in Metropolitan and Nonmetropolitan Counties — United States, 2016
}

\author{
Elizabeth A. Lundeen, $\mathrm{PhD}^{1}$; Sohyun Park, $\mathrm{PhD}^{1}$; Liping Pan, $\mathrm{MD}^{1}$; Terry O’Toole, $\mathrm{PhD}^{1}$; Kevin Matthews, $\mathrm{PhD}^{2}$; Heidi M. Blanck, $\mathrm{PhD}^{1}$
}

Approximately 46 million persons (14\%) in the United States live in nonmetropolitan counties.* Compared with metropolitan residents, nonmetropolitan residents have a higher prevalence of obesity-associated chronic diseases such as diabetes (1), coronary heart disease (1), and arthritis (2). The 2005-2008 National Health and Nutrition Examination Survey (NHANES) found a significantly higher obesity prevalence among adults in nonmetropolitan $(39.6 \%)$ than in metropolitan (33.4\%) counties (3). However, this difference has not been examined by state. Therefore, CDC examined state-level 2016 Behavioral Risk Factor Surveillance System (BRFSS) data and found that the prevalence of obesity (body mass index $[\mathrm{BMI}] \geq 30 \mathrm{~kg} / \mathrm{m}^{2}$ ) was $34.2 \%$ among U.S. adults living in nonmetropolitan counties and $28.7 \%$ among those living in metropolitan counties $(\mathrm{p}<0.001)$. Obesity prevalence was significantly higher among nonmetropolitan county residents than among metropolitan county residents in all U.S. Census regions, with the largest absolute difference in the South (5.6 percentage points) and Northeast (5.4 percentage points). In 24 of 47 states, obesity prevalence was significantly higher among persons in nonmetropolitan counties than among those in metropolitan counties; only in Wyoming was obesity prevalence higher among metropolitan county residents than among nonmetropolitan county residents. Both metropolitan and nonmetropolitan counties can address obesity through a variety of policy and environmental strategies to increase access to healthier foods and opportunities for physical activity (4).

BRFSS is a state-based, random-digit-dialed telephone survey of U.S. adults aged $\geq 18$ years, conducted annually by $\mathrm{CDC}$ and state and territorial health departments to monitor health conditions and related behaviors. ${ }^{\dagger}$ BRFSS uses multistage, stratified sampling to select a representative sample

\footnotetext{
*https://www.ers.usda.gov/webdocs/publications/80894/eib-162.pdf?v=42684.

${ }^{\dagger}$ https://www.cdc.gov/brfss/annual_data/annual_2016.html.
}

of the noninstitutionalized adult population in 50 states, the District of Columbia (DC), and selected U.S. territories. In 2016, using combined landline and cell phone data across all states, the median response rate was $47.0 \%$, which was calculated using rates from the American Association of Public Opinion Research. ${ }^{\S}$ Self-reported weight and height were used to calculate BMI (weight $[\mathrm{kg}] /$ height $[\mathrm{m}]^{2}$ ); obesity was defined as BMI $\geq 30 \mathrm{~kg} / \mathrm{m}^{2}$. Among 477,665 respondents, 39,186 (8.2\%) were excluded, including 36,848 with missing BMI values and 2,338 with implausible BMI values, leaving a final analytic sample of 438,479 adults from 50 states and DC. Unadjusted obesity prevalence is presented overall and

\$https://www.cdc.gov/brfss/annual_data/2016/pdf/2016-sdqr.pdf.
9 https://www.nhlbi.nih.gov/files/docs/guidelines/ob_gdlns.pdf.

\section{INSIDE}

659 Outbreak of E. coli O157:H7 Infections Associated with Exposure to Animal Manure in a Rural Community — Arizona and Utah, June-July 2017

663 Peer-Delivered Linkage Case Management and Same-Day ART Initiation for Men and Young Persons with HIV Infection — Eswatini, 2015-2017

668 Notes from the Field: Carbapenemase-Producing Carbapenem-Resistant Enterobacteriaceae from Less Common Enterobacteriaceae Genera — United States, 2014-2017

670 Notes from the Field: Contact Investigation for an Infant with Congenital Tuberculosis Infection North Carolina, 2016

672 QuickStats

Continuing Education examination available at https://www.cdc.gov/mmwr/cme/conted_info.html\#weekly.

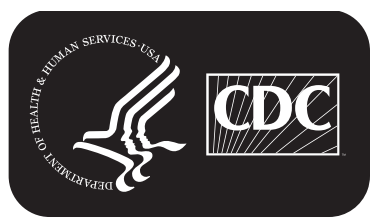

U.S. Department of Health and Human Services Centers for Disease Control and Prevention 
by sociodemographic characteristics (age, sex, race/ethnicity, education, income, and employment status), state, and four U.S. Census regions and nine divisions: Northeast region (New England and Middle Atlantic divisions), Midwest region (East North Central and West North Central divisions), South region (South Atlantic, East South Central, and West South Central divisions), and West region (Mountain and Pacific divisions).**

Using 2010 Census data, CDC's National Center for Health Statistics (NCHS) developed an Urban-Rural Classification Scheme for Counties, ${ }^{\dagger \dagger}$ which specified six county types; for this analysis, to ensure sufficient sample size for regional and state-level comparisons, counties were collapsed into two categories: metropolitan (large central metro, large fringe metro, medium metro, and small metro) and nonmetropolitan (micropolitan and noncore). In this analysis, the nonmetropolitan designation was used to classify counties with small populations $(<50,000)$. Rhode Island, New Jersey, Delaware, and DC do not have nonmetropolitan counties; for these jurisdictions, obesity prevalence was calculated for adults living in metropolitan counties only. U.S. territories were excluded because the NCHS classification scheme does not include them. Unadjusted obesity prevalence was stratified by metropolitan and nonmetropolitan status. Differences in obesity prevalence between adults living in metropolitan and nonmetropolitan counties were examined using multivariable logistic regression,

\footnotetext{
** https://www.census.gov/geo/reference/gtc/gtc_census_divreg.html.

$\dagger^{\dagger}$ https://www.cdc.gov/nchs/data_access/urban_rural.htm.
}

controlling for age, sex, and race/ethnicity within levels of the sociodemographic characteristics, states, and Census regions and divisions (statistically significant at $\mathrm{p}<0.05$ ). All analyses accounted for complex survey design and sampling weights.

In 2016, overall obesity prevalence was $29.6 \%$ and was highest among persons residing in the South $(32.0 \%)$ and Midwest (31.4\%) regions and the East South Central (35.3\%) and West South Central (33.9\%) divisions (Table 1). Overall, obesity prevalence was significantly higher among adults living in nonmetropolitan counties (34.2\%) than among those living metropolitan counties $(28.7 \%)(p<0.001)$, and the same was found in all Census regions and Census divisions. Among Census regions, the largest difference in obesity prevalence between persons living in nonmetropolitan and metropolitan counties was in the South (5.6 percentage points) and Northeast (5.4 percentage points); among Census divisions, the largest difference in obesity prevalence between nonmetropolitan and metropolitan residents was in the Middle Atlantic division (6.6 percentage points). Obesity prevalence was also significantly higher among nonmetropolitan county residents than among metropolitan county residents for all sociodemographic categories except Hispanics and persons with less than a high school education.

Among adults living in nonmetropolitan counties, obesity prevalence ranged from $20.8 \%$ in Colorado to $39.1 \%$ in Louisiana; among those living in metropolitan counties, prevalence ranged from $22.5 \%$ in Colorado to $36.9 \%$ in West Virginia. (Table 2). In 24 (51\%) of the 47 states with both

The MMWR series of publications is published by the Center for Surveillance, Epidemiology, and Laboratory Services, Centers for Disease Control and Prevention (CDC), U.S. Department of Health and Human Services, Atlanta, GA 30329-4027.

Suggested citation: [Author names; first three, then et al., if more than six.] [Report title]. MMWR Morb Mortal Wkly Rep 2018;67:[inclusive page numbers].

\section{Centers for Disease Control and Prevention Robert R. Redfield, MD, Director \\ Anne Schuchat, MD, Principal Deputy Director \\ Leslie Dauphin, PhD, Acting Associate Director for Science \\ Joanne Cono, MD, ScM, Director, Office of Science Quality \\ Chesley L. Richards, MD, MPH, Deputy Director for Public Health Scientific Services \\ Michael F. Iademarco, MD, MPH, Director, Center for Surveillance, Epidemiology, and Laboratory Services \\ MMWR Editorial and Production Staff (Weekly)}

Charlotte K. Kent, PhD, MPH, Acting Editor in Chief, Executive Editor

Jacqueline Gindler, MD, Editor

Mary Dott, MD, MPH, Online Editor

Teresa F. Rutledge, Managing Editor

Douglas W. Weatherwax, Lead Technical Writer-Editor

Glenn Damon, Soumya Dunworth, PhD, Teresa M. Hood, MS, Technical Writer-Editors
Martha F. Boyd, Lead Visual Information Specialist Maureen A. Leahy, Julia C. Martinroe, Stephen R. Spriggs, Tong Yang, Visual Information Specialists

Quang M. Doan, MBA, Phyllis H. King,

Terraye M. Starr, Moua Yang,

Information Technology Specialists

\author{
MMWR Editorial Board \\ Timothy F. Jones, MD, Chairman \\ William E. Halperin, MD, DrPH, MPH \\ King K. Holmes, MD, PhD \\ Robin Ikeda, MD, MPH \\ Phyllis Meadows, PhD, MSN, RN \\ Jewel Mullen, MD, MPH, MPA
}

Matthew L. Boulton, MD, MPH

Virginia A. Caine, MD

Katherine Lyon Daniel, PhD

Jonathan E. Fielding, MD, MPH, MBA

David W. Fleming, MD
Jeff Niederdeppe, PhD

Patricia Quinlisk, MD, MPH

Patrick L. Remington, MD, MPH

Carlos Roig, MS, MA

William Schaffner, MD 
TABLE 1. Prevalence of self-reported obesity among adults (aged $\geq 18$ years) by respondent characteristics and metropolitan/nonmetropolitan status - Behavioral Risk Factor Surveillance System, 50 states and the District of Columbia, 2016

\begin{tabular}{|c|c|c|c|c|}
\hline \multirow[b]{2}{*}{ Characteristic } & \multirow[b]{2}{*}{ No. of respondents } & \multicolumn{3}{|c|}{ Unadjusted adult obesity prevalence-weighted $\%(95 \% \mathrm{Cl})^{*}$} \\
\hline & & Total & Metropolitan ${ }^{\dagger}$ & Nonmetropolitan ${ }^{\dagger}$ \\
\hline Total & 438,479 & $29.6(29.3-29.8)$ & $28.7(28.4-29.0)^{\S}$ & $34.2(33.6-34.8)^{\S}$ \\
\hline $\begin{array}{l}\text { Age group (yrs) } \\
18-24 \\
25-34 \\
35-44 \\
45-54 \\
55-64 \\
\geq 65\end{array}$ & $\begin{array}{r}23,734 \\
42,706 \\
48,951 \\
68,854 \\
96,566 \\
157,668\end{array}$ & $\begin{array}{l}17.3(16.5-18.1) \\
27.2(26.5-27.9) \\
33.1(32.3-33.8) \\
35.1(34.4-35.8) \\
34.2(33.6-34.8) \\
28.0(27.5-28.5)\end{array}$ & $\begin{array}{l}16.5(15.6-17.4)^{\S} \\
26.4(25.6-27.2)^{\S} \\
32.0(31.2-32.9)^{\S} \\
34.0(33.2-34.8)^{\S} \\
33.4(32.7-34.1)^{\S} \\
27.5(26.9-28.1)^{\S}\end{array}$ & $\begin{array}{l}22.2(20.3-24.2)^{\S} \\
32.5(30.8-34.3)^{\S} \\
39.6(38.0-41.2)^{\S} \\
40.8(39.4-42.3)^{\S} \\
38.0(36.9-39.2)^{\S} \\
30.1(29.3-31.0)^{\S}\end{array}$ \\
\hline $\begin{array}{l}\text { Sex } \\
\text { Male } \\
\text { Female }\end{array}$ & $\begin{array}{l}198,440 \\
240,000\end{array}$ & $\begin{array}{l}29.6(29.2-30.0) \\
29.5(29.1-29.9)\end{array}$ & $\begin{array}{l}28.8(28.3-29.2)^{\S} \\
28.7(28.2-29.1)^{\S}\end{array}$ & $\begin{array}{l}34.4(33.6-35.2)^{\S} \\
34.0(33.2-34.8)^{\S}\end{array}$ \\
\hline $\begin{array}{l}\text { Race/Ethnicity }{ }^{\mathfrak{f}, * *} \\
\text { White, non-Hispanic } \\
\text { Black, non-Hispanic } \\
\text { Hispanic, any race } \\
\text { Other, non-Hispanic }\end{array}$ & $\begin{array}{r}341,192 \\
35,091 \\
28,666 \\
26,954\end{array}$ & $\begin{array}{l}28.6(28.3-28.9) \\
38.3(37.4-39.3) \\
33.1(32.1-34.1) \\
18.2(17.3-19.2)\end{array}$ & $\begin{array}{l}27.5(27.2-27.9) \S \\
37.7(36.7-38.7)^{\S} \\
32.9(31.9-33.9) \\
16.8(15.8-17.8)^{\S}\end{array}$ & $\begin{array}{l}33.2(32.6-33.8)^{\S} \\
44.2(41.7-46.7)^{\S} \\
36.0(32.6-39.5) \\
33.2(31.2-35.3)^{\S}\end{array}$ \\
\hline $\begin{array}{l}\text { Education }{ }^{\uparrow, * *} \\
<\text { High school } \\
\text { High school } \\
\text { Some college } \\
\text { College graduate }\end{array}$ & $\begin{array}{r}32,325 \\
123,241 \\
120,735 \\
161,309\end{array}$ & $\begin{array}{l}35.5(34.5-36.5) \\
32.3(31.8-32.8) \\
31.0(30.5-31.5) \\
22.2(21.9-22.6)\end{array}$ & $\begin{array}{l}35.4(34.3-36.6) \\
31.5(30.9-32.1)^{\S} \\
30.3(29.7-30.9)^{\S} \\
21.5(21.1-21.9)^{\S}\end{array}$ & $\begin{array}{l}35.9(34.0-37.8) \\
35.6(34.7-36.5)^{\S} \\
34.7(33.7-35.7)^{\S} \\
28.8(27.9-29.7)^{\S}\end{array}$ \\
\hline $\begin{array}{l}\text { Annual household in } \\
<\$ 25,000 \\
\$ 25,000-49,999 \\
\$ 50,000-74,999 \\
\geq \$ 75,000\end{array}$ & $\begin{array}{r}99,244 \\
95,553 \\
61,211 \\
120,901\end{array}$ & $\begin{array}{l}34.1(33.5-34.7) \\
31.9(31.3-32.6) \\
31.1(30.3-31.8) \\
25.4(24.9-25.9)\end{array}$ & $\begin{array}{l}33.4(32.7-34.2)^{\S} \\
31.1(30.3-31.8)^{\S} \\
30.2(29.4-31.1)^{\S} \\
24.8(24.3-25.3)^{\S}\end{array}$ & $\begin{array}{l}37.1(35.9-38.2)^{\S} \\
35.9(34.7-37.1)^{\S} \\
35.4(34.0-36.8)^{\S} \\
30.9(29.8-32.1)^{\S}\end{array}$ \\
\hline $\begin{array}{l}\text { Employment status } \\
\text { Employed } \\
\text { Out of work } \\
\text { Homemaker } \\
\text { Student } \\
\text { Retired } \\
\text { Unable to work }\end{array}$ & $\begin{array}{r}215,226 \\
17,009 \\
22,372 \\
11,277 \\
136,638 \\
33,534\end{array}$ & $\begin{array}{l}29.0(28.6-29.4) \\
32.9(31.6-34.3) \\
29.0(27.7-30.3) \\
15.2(14.1-16.3) \\
29.1(28.5-29.6) \\
45.8(44.8-46.9)\end{array}$ & $\begin{array}{l}28.2(27.8-28.6)^{\S} \\
32.4(30.9-34.0)^{\S} \\
28.4(27.0-29.9)^{\S} \\
14.8(13.6-16.0)^{\S} \\
28.6(28.0-29.2)^{\S} \\
45.5(44.2-46.8)^{\S}\end{array}$ & $\begin{array}{l}34.1(33.3-34.9)^{\S} \\
35.8(33.1-38.7)^{\S} \\
32.0(29.5-34.7)^{\S} \\
18.8(16.2-21.7)^{\S} \\
31.2(30.3-32.2)^{\S} \\
47.1(45.2-49.1)^{\S}\end{array}$ \\
\hline $\begin{array}{l}\text { Census region }{ }^{\natural,+\dagger} \\
\text { Northeast } \\
\text { Midwest } \\
\text { South } \\
\text { West }\end{array}$ & $\begin{array}{r}88,335 \\
106,697 \\
146,919 \\
96,528\end{array}$ & $\begin{array}{l}26.9(26.3-27.5) \\
31.4(30.9-31.9) \\
32.0(31.5-32.5) \\
26.0(25.4-26.6)\end{array}$ & $\begin{array}{l}26.4(25.8-27.0)^{\S} \\
30.5(29.9-31.2)^{\S} \\
31.0(30.4-31.6)^{\S} \\
25.7(25.1-26.4)^{\S}\end{array}$ & $\begin{array}{l}31.8(30.4-33.2)^{\S} \\
34.2(33.3-35.1)^{\S} \\
36.6(35.6-37.6)^{\S} \\
28.6(27.5-29.7)^{\S}\end{array}$ \\
\hline $\begin{array}{l}\text { Census division }{ }^{\text {f,t† }} \\
\text { New England } \\
\text { Middle Atlantic } \\
\text { East North Central } \\
\text { West North Central } \\
\text { South Atlantic } \\
\text { East South Central } \\
\text { West South Central } \\
\text { Mountain } \\
\text { Pacific }\end{array}$ & $\begin{array}{l}43,889 \\
44,446 \\
42,215 \\
64,482 \\
93,367 \\
26,587 \\
26,965 \\
57,788 \\
38,740\end{array}$ & $\begin{array}{l}25.4(24.7-26.1) \\
27.4(26.7-28.2) \\
31.8(31.1-32.5) \\
30.6(30.0-31.2) \\
29.9(29.3-30.4) \\
35.3(34.4-36.2) \\
33.9(32.7-35.2) \\
26.2(25.6-26.8) \\
25.9(25.0-26.7)\end{array}$ & $\begin{array}{l}25.0(24.2-25.8)^{\S} \\
26.9(26.1-27.7)^{\S} \\
31.0(30.2-31.8)^{\S} \\
29.3(28.5-30.1)^{\S} \\
29.1(28.5-29.7)^{\S} \\
34.5(33.3-35.6)^{\S} \\
33.1(31.7-34.5)^{\S} \\
26.0(25.3-26.7)^{\S} \\
25.6(24.7-26.4)^{\S}\end{array}$ & $\begin{array}{l}28.7(27.4-30.0)^{\S} \\
33.5(31.5-35.6)^{\S} \\
34.9(33.5-36.3)^{\S} \\
33.3(32.4-34.2)^{\S} \\
35.3(33.9-36.7)^{\S} \\
36.9(35.6-38.1)^{\S} \\
37.8(35.4-40.3)^{\S} \\
27.2(26.3-28.1)^{\S} \\
30.3(28.1-32.6)^{\S}\end{array}$ \\
\hline
\end{tabular}

Abbreviation: $\mathrm{Cl}=$ confidence interval.

* Obesity defined as having a body mass index $\geq 30 \mathrm{~kg} / \mathrm{m} 2$ determined by self-reported weight and height.

${ }^{\dagger}$ Based on National Center for Health Statistics Urban-Rural Classification Scheme for Counties. Metropolitan includes large central metro, large fringe metro, medium metro, and small metro categories. Nonmetropolitan includes micropolitan and noncore categories.

$\S$ Significant difference in the prevalence of obesity between metropolitan and nonmetropolitan areas at the $p<0.05$ level. Determined using multivariable logistic regression within levels of the sociodemographic and geographic characteristics to control for age, sex, and race/ethnicity.

I Significant difference in the prevalence of obesity across levels of the characteristic at the $p<0.05$ level using Chi-square test.

** Missing data: sex $(n=39 ; 0.009 \%)$, race/ethnicity $(n=6,576 ; 1.5 \%)$, education $(n=869 ; 0.2 \%)$, income $(n=61,570 ; 14.0 \%)$, and employment status $(n=2,423 ; 0.6 \%)$.

${ }^{+\dagger}$ The United States Census Bureau defines four census regions and nine census divisions: Northeast region (New England and Middle Atlantic divisions), Midwest region (East North Central and West North Central divisions), Southern region (South Atlantic, East South Central, and West South Central divisions), and Western region (Mountain and Pacific divisions). 
metropolitan and nonmetropolitan counties, obesity prevalence was significantly higher among adults living in nonmetropolitan counties than among those living in metropolitan counties; in $22(47 \%)$ states, no difference was observed. Wyoming was the only state where obesity prevalence was significantly higher among metropolitan county residents $(32.8 \%)$ than among nonmetropolitan residents $(25.4 \% ; \mathrm{p}=0.002)$.

\section{Discussion}

In this study, obesity prevalence was significantly higher among adults living in nonmetropolitan counties than among those living in metropolitan counties, overall, in all Census regions, all Census divisions, and in approximately half of states with both county types. Across regions and divisions, this

TABLE 2. Prevalence of self-reported obesity among adults (aged $\geq 18$ years) by state and metropolitan/nonmetropolitan status Behavioral Risk Factor Surveillance System, 50 states and the District of Columbia, 2016

\begin{tabular}{|c|c|c|c|}
\hline \multirow{2}{*}{$\begin{array}{l}\text { Census } \\
\text { division }^{\dagger} / \text { State }\end{array}$} & \multirow{2}{*}{$\begin{array}{l}\text { No. of } \\
\text { respondents }\end{array}$} & \multicolumn{2}{|c|}{$\begin{array}{c}\text { Unadjusted adult obesity prevalence- } \\
\text { weighted } \%(95 \% \mathrm{Cl})^{*}\end{array}$} \\
\hline & & Metropolitan $\S$ & Nonmetropolitan $\S$ \\
\hline \multicolumn{4}{|l|}{ New England } \\
\hline Connecticut & 9,960 & $25.9(24.7-27.1)$ & $28.1(22.7-34.2)$ \\
\hline Maine & 9,554 & $29.3(27.3-31.3)$ & $30.9(29.1-32.7)$ \\
\hline Massachusetts & 7,480 & $23.6(22.2-24.9)$ & $24.4(16.9-34.0)$ \\
\hline New Hampshire & 5,888 & $26.0(23.8-28.2)$ & $27.6(25.4-29.9)$ \\
\hline Rhode Island & 4,936 & $26.6(24.9-28.4)$ & -9 \\
\hline Vermont & 6,071 & $24.1(21.3-27.1)^{* *}$ & $28.7(26.9-30.6)^{* *}$ \\
\hline \multicolumn{4}{|l|}{ Middle Atlantic } \\
\hline New Jersey & 6,810 & $27.4(25.7-29.1)$ & - \\
\hline New York & 31,269 & $24.9(23.9-26.0)^{* *}$ & $33.0(31.6-34.5)^{* *}$ \\
\hline Pennsylvania & 6,367 & $29.7(28.1-31.4)^{* *}$ & $33.9(30.4-37.5)^{* *}$ \\
\hline \multicolumn{4}{|c|}{ East North Central } \\
\hline Illinois & 4,518 & $31.0(29.2-32.9)^{* *}$ & $35.7(31.0-40.6)^{* * *}$ \\
\hline Indiana & 10,319 & $32.0(30.6-33.5)$ & $33.9(31.3-36.7)$ \\
\hline Michigan & 11,130 & $31.6(30.4-32.9)^{* *}$ & $36.0(33.7-38.5)^{* *}$ \\
\hline Ohio & 11,455 & $30.7(29.2-32.3)^{* * *}$ & $34.4(32.1-36.8)^{* *}$ \\
\hline Wisconsin & 4,793 & $29.1(27.0-31.3)^{* *}$ & $34.4(31.6-37.3)^{* *}$ \\
\hline \multicolumn{4}{|c|}{ West North Central } \\
\hline lowa & 6,645 & $31.4(29.4-33.5)$ & 32.7 (30.7-34.8) \\
\hline Kansas & 10,947 & $29.9(28.5-31.3)^{* *}$ & $33.7(32.0-35.5)^{* *}$ \\
\hline Minnesota & 15,420 & $26.5(25.6-27.5)^{* *}$ & $31.7(30.1-33.2)^{* *}$ \\
\hline Missouri & 6,578 & $30.5(28.4-32.6)^{* *}$ & $34.9(32.1-37.9)^{* *}$ \\
\hline Nebraska & 14,173 & $30.8(29.1-32.6)^{* *}$ & $34.2(32.9-35.5)^{* *}$ \\
\hline North Dakota & 5,348 & $30.5(28.2-32.9)$ & $33.4(31.2-35.6)$ \\
\hline South Dakota & 5,371 & $27.0(23.9-30.5)^{* *}$ & $31.8(29.2-34.5)^{* * *}$ \\
\hline \multicolumn{4}{|l|}{ South Atlantic } \\
\hline Delaware & 3,702 & $30.7(28.7-32.8)$ & - \\
\hline District of & 3,479 & $22.6(20.9-24.3)$ & 一ा \\
\hline \multicolumn{4}{|l|}{ Columbia } \\
\hline Florida & 33,186 & $27.2(26.1-28.2)^{* * *}$ & $34.9(32.6-37.2)^{* *}$ \\
\hline Georgia & 4,884 & $30.8(28.9-32.8)$ & $34.0(30.3-37.9)$ \\
\hline Maryland & 16,701 & $29.8(28.7-30.9)^{* *}$ & $35.1(32.0-38.3)^{* *}$ \\
\hline North Carolina & 5,984 & $31.1(29.5-32.9)$ & $34.1(31.4-37.0)$ \\
\hline South Carolina & 10,503 & $31.2(29.8-32.7)^{* * *}$ & $37.8(35.1-40.6)^{* * *}$ \\
\hline Virginia & 8,293 & $27.7(26.3-29.1)^{* *}$ & $36.1(33.2-39.1)^{* *}$ \\
\hline West Virginia & 6,635 & $36.9(35.2-38.7)$ & $38.8(36.6-41.0)$ \\
\hline \multicolumn{4}{|c|}{ East South Central } \\
\hline Alabama & 6,526 & $35.6(33.8-37.5)$ & $36.0(33.1-38.9)$ \\
\hline Kentucky & 9,583 & $32.1(30.2-34.0)^{* *}$ & $36.9(34.7-39.2)^{* *}$ \\
\hline
\end{tabular}

disparity in obesity prevalence was highest in the South and Northeast regions and the Middle Atlantic division. With the exception of Hispanics and persons with less than a high school education, the higher obesity prevalence among nonmetropolitan residents was observed in all sociodemographic groups.

These findings are consistent with those previously reported using 2005-2008 NHANES data, which documented higher overall obesity prevalence among adults living in nonmetropolitan versus metropolitan counties of the United States (3), and expand the understanding of this health disparity by highlighting differences across states and regions. Research has documented differences between adults living in nonmetropolitan and metropolitan counties in health behaviors and community factors, which could influence obesity risk (5-7).

TABLE 2. (Continued) Prevalence of self-reported obesity among adults (aged $\geq 18$ years) by state and metropolitan/nonmetropolitan status Behavioral Risk Factor Surveillance System, 50 states and the District of Columbia, 2016

\begin{tabular}{|c|c|c|c|}
\hline \multirow{2}{*}{$\begin{array}{l}\text { Census } \\
\text { division }{ }^{\dagger} / \text { State }\end{array}$} & \multirow{2}{*}{$\begin{array}{l}\text { No. of } \\
\text { respondents }\end{array}$} & \multicolumn{2}{|c|}{$\begin{array}{c}\text { Unadjusted adult obesity prevalence- } \\
\text { weighted } \%(95 \% \mathrm{CI})^{*}\end{array}$} \\
\hline & & Metropolitan $§$ & Nonmetropolitan $§$ \\
\hline Mississippi & 4,821 & 36.5 (33.4-39.7) & $37.9(35.7-40.1)$ \\
\hline Tennessee & 5,657 & $34.3(32.1-36.6)$ & $36.4(33.6-39.3)$ \\
\hline \multicolumn{4}{|c|}{ West South Central } \\
\hline Arkansas & 4,859 & $35.4(32.2-38.8)$ & 36.1 (32.6-39.7) \\
\hline Louisiana & 4,868 & 34.8 (32.5-37.3) & 39.1 (34.7-43.7) \\
\hline Oklahoma & 6,449 & $30.8(28.8-32.8)^{* * *}$ & $36.3(33.9-38.8)^{* *}$ \\
\hline Texas & 10,789 & $32.9(31.0-34.8)^{* *}$ & $38.7(34.3-43.2)^{* * *}$ \\
\hline \multicolumn{4}{|l|}{ Mountain } \\
\hline Arizona & 10,033 & $28.8(27.2-30.4)$ & 33.6 (29.1-38.4) \\
\hline Colorado & 13,637 & $22.5(21.5-23.5)$ & $20.8(19.0-22.8)$ \\
\hline Idaho & 4,880 & 26.3 (23.9-28.8) & $29.6(27.0-32.4)$ \\
\hline Montana & 5,483 & $25.9(23.1-29.0)$ & $25.3(23.3-27.3)$ \\
\hline Nevada & 3,981 & $25.1(23.1-27.3)^{* *}$ & $32.1(28.6-35.9)^{* *}$ \\
\hline New Mexico & 5,531 & $27.0(24.7-29.4)^{* *}$ & $31.1(28.7-33.6)^{* *}$ \\
\hline Utah & 10,043 & $25.4(24.2-26.7)$ & $24.9(22.7-27.2)$ \\
\hline Wyoming & 4,200 & $32.8(29.0-36.9)^{* *}$ & $25.4(23.1-27.8)^{* * *}$ \\
\hline \multicolumn{4}{|l|}{ Pacific } \\
\hline Alaska & 2,739 & $30.9(27.1-35.0)$ & $32.4(28.8-36.4)$ \\
\hline California & 10,352 & $25.0(24.0-26.1)$ & $24.2(19.2-30.0)$ \\
\hline Hawaii & 7,659 & $23.3(21.8-24.9)^{* *}$ & $26.1(23.5-28.8)^{* * *}$ \\
\hline Oregon & 5,000 & $27.4(25.8-29.1)^{* *}$ & $35.1(31.5-38.8)^{* *}$ \\
\hline Washington & 12,990 & $27.8(26.8-28.9)^{* * *}$ & $35.3(32.3-38.4)^{* *}$ \\
\hline
\end{tabular}

Abbreviation: $\mathrm{Cl}=$ confidence interval.

* Obesity defined as having a body mass index $\geq 30 \mathrm{~kg} / \mathrm{m} 2$, determined by self-reported weight and height.

† The United States Census Bureau defines nine census divisions within four regions: Northeast region (New England and Middle Atlantic divisions), Midwest region (East North Central and West North Central divisions), Southern region (South Atlantic, East South Central, and West South Central divisions), and Western region (Mountain and Pacific divisions).

$\S$ Based on National Center for Health Statistics Urban-Rural Classification Scheme for Counties. Metropolitan includes large central metro, large fringe metro, medium metro, and small metro categories. Nonmetropolitan includes micropolitan and noncore categories.

"Data not available because state does not have counties in the nonmetropolitan classification.

** Significant difference in the prevalence of obesity between metropolitan and nonmetropolitan areas at the $p<0.05$ level. Within states, differences in obesity prevalence between metropolitan and nonmetropolitan areas were determined using multivariable logistic regression, controlling for age, sex, and race/ethnicity 
An analysis of 2013 BRFSS data found that adults living in U.S. nonmetropolitan counties were less physically active and less likely to meet physical activity recommendations than their metropolitan counterparts (5). Data from 2011 indicated that across all regions, adults living in rural areas were less likely to have access to healthier food retailers (supermarkets, large grocery stores, and fruit/vegetable specialty stores) than were those living in urban areas (G). In addition, several social determinants of health that are risk factors for obesity, such as persistent poverty and food insecurity ( 7$)$, are more prevalent in rural than in urban areas. $\$ \$ \$, \mathbf{s g}$

In this analysis, the highest obesity prevalence and the greatest disparity in prevalence between persons living in nonmetropolitan and metropolitan counties were in the South Census region. One possible contributing factor is the high rate of persistent poverty in the South, which also is affected by the largest difference in poverty rate between metropolitan and nonmetropolitan county residents. 99

The findings in this report are subject to at least two limitations. First, data are self-reported, and self-reported weight and height data underestimate BMI values, particularly among persons with a higher BMI (8). It is not known whether self-reporting bias is comparable across regions and between metropolitan and nonmetropolitan counties. Second, to ensure sufficient sample size for regional and state-level comparisons, the nonmetropolitan classification was used to designate counties with small populations ( $<50,000$ persons). The literature on rural obesity disparities and prevention strategies uses various methods to define rural areas, some of which might differ in population size from the nonmetropolitan designation used in this paper.

CDC recommends 24 obesity-prevention policy and environmental strategies (4). Two systematic reviews summarized the relevance and effectiveness of these strategies in rural areas and identified how these strategies could be adapted for rural settings $(9,10)$. One nutrition-related obesity prevention strategy, increasing the availability of healthier food and beverage choices, is challenging to implement in rural areas because of the long distances between food suppliers and retailers and between retailers and consumers, which can influence food cost and the availability of fresh foods. Approaches to overcoming this challenge include strengthening networks between food producers, distributors, and retail food outlets, as well as reducing the distance customers need to travel, for example, by increasing access to nearby farmers' markets (9). The 2018 CDC State Indicator Report on Fruits and Vegetables also

\footnotetext{
$\overline{\$ \$}$ https://www.ers.usda.gov/webdocs/publications/79761/err-215.pdf?v=42636.

99 https://www.ers.usda.gov/topics/rural-economy-population/ rural-poverty-well-being/.
}

\section{Summary}

What is already known about this topic?

National estimates from a decade ago found a higher prevalence of obesity among adults living in nonmetropolitan counties than among those living in metropolitan counties.

What is added by this report?

Analysis of 2016 Behavioral Risk Factor Surveillance System data found a higher obesity prevalence among adults in nonmetropolitan counties than among those in metropolitan counties. The greatest differences in obesity prevalence between nonmetropolitan and metropolitan residents were in the South (5.6 percentage points) and Northeast (5.4 percentage points).

What are the implications for public health practice?

Both nonmetropolitan and metropolitan counties can address obesity through a variety of policy and environmental strategies to increase access to healthier foods and opportunities for physical activity.

highlights approaches to increase the purchase, supply, and demand of fruits and vegetables in states and communities across the United States. ${ }^{* * *}$ Other approaches include working with schools and worksites to develop nutrition-related policies and forming strong partnerships with groups such as the Cooperative Extension Service to promote federal food and nutrition assistance program benefits (9).

Strategies to increase physical activity in rural areas should take into consideration geographic dispersion, transportation challenges, and limitations on community resources that might not be present in urban areas (10). Strategies that have been implemented in rural settings include improving community access to public buildings (e.g., school facilities) after hours for physical activity purposes; improving infrastructure and land use design to support walking and other physical activity (e.g., bicycle paths, paved sidewalks, and outdoor public recreation facilities); promoting existing places for physical activity with improved signage; enhancing physical education in schools; and implementing worksite policies to promote physical activity (10). The data in this report can serve as a resource for states seeking to reduce obesity disparities in nonmetropolitan counties through strategies to increase physical activity and healthier eating.

\section{Acknowledgments}

William Garvin, Division of Population Health, National Center for Chronic Disease Prevention and Health Promotion, CDC; state and DC BRFSS staff members. *** https://www.cdc.gov/nutrition/data-statistics/2018-state-indicator-report-
fruits-vegetables.html. 


\section{Conflict of Interest}

No conflicts of interest were reported.

${ }^{1}$ Division of Nutrition, Physical Activity, and Obesity, National Center for Chronic Disease Prevention and Health Promotion, CDC; ${ }^{2}$ Division of Population Health, National Center for Chronic Disease Prevention and Health Promotion, CDC.

Corresponding author: Elizabeth A. Lundeen, elundeen@cdc.gov, 770-488-6517.

\section{References}

1. O'Connor A, Wellenius G. Rural-urban disparities in the prevalence of diabetes and coronary heart disease. Public Health 2012;126:813-20. https://doi.org/10.1016/j.puhe.2012.05.029

2. Boring MA, Hootman JM, Liu Y, et al. Prevalence of arthritis and arthritis-attributable activity limitation by urban-rural county classification-United States, 2015. MMWR Morb Mortal Wkly Rep 2017;66:527-32. https://doi.org/10.15585/mmwr.mm6620a2

3. Befort CA, Nazir N, Perri MG. Prevalence of obesity among adults from rural and urban areas of the United States: findings from NHANES (2005-2008). J Rural Health 2012;28:392-7. https://doi. org/10.1111/j.1748-0361.2012.00411.x
4. Khan LK, Sobush K, Keener D, et al. Recommended community strategies and measurements to prevent obesity in the United States. MMWR Recomm Rep 2009;58(No. RR-7).

5. Matthews KA, Croft JB, Liu Y, et al. Health-related behaviors by urbanrural county classification-United States, 2013. MMWR Surveill Summ 2017;66(No. SS-5). https://doi.org/10.15585/mmwr.ss6605a1

6. Grimm KA, Moore LV, Scanlon KS. Access to healthier food retailersUnited States, 2011. MMWR Suppl 2013;62:20-6.

7. Bhattacharya J, Currie J, Haider S. Poverty, food insecurity, and nutritional outcomes in children and adults. J Health Econ 2004;23:839-62. https://doi.org/10.1016/j.jhealeco.2003.12.008

8. Stommel M, Schoenborn CA. Accuracy and usefulness of BMI measures based on self-reported weight and height: findings from the NHANES \& NHIS 2001-2006. BMC Public Health 2009;9:421. https://doi. org/10.1186/1471-2458-9-421

9. Calancie L, Leeman J, Jilcott Pitts SB, et al. Nutrition-related policy and environmental strategies to prevent obesity in rural communities: a systematic review of the literature, 2002-2013. Prev Chronic Dis 2015;12:140540. https://doi.org/10.5888/pcd12.140540

10. Umstattd Meyer MR, Perry CK, Sumrall JC, et al. Physical activityrelated policy and environmental strategies to prevent obesity in rural communities: a systematic review of the literature, 2002-2013. Prev Chronic Dis 2016;13:150406. https://doi.org/10.5888/pcd13.150406 